\title{
Comparing risk-adjusted outcomes from out-of-hospital cardiac arrest across Australia and New Zealand
}

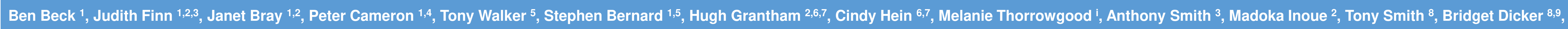
Andy Swain ${ }^{10}$, Emma Bosley ${ }^{11,12}$, Malcolm Johnston-Leek ${ }^{13}$, Gavin D. Perkins ${ }^{14}$, Graham Nichol ${ }^{15}$, Karen Smith ${ }^{1,5,16}$, on behalf of the Aus-ROC Steering Committee

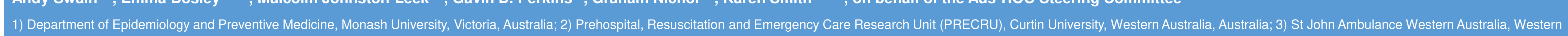

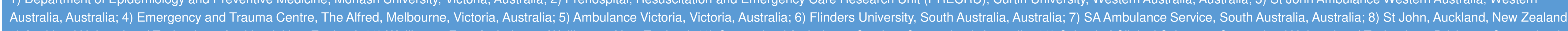

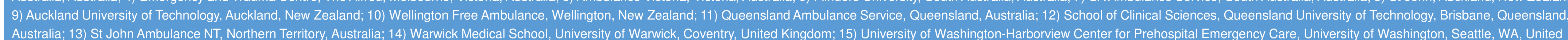
Australia; 13) St John Ambulance NT, Northern Territory, Australia; 14) Warwick Medical School, University of Warwick,
States of America; 16) Department of Community Emergency Health and Paramedic Practice, Monash University

\section{PURPOSE}

The aim of this study was to quantify the complex relationship between key Utstein variables to inform improvements in benchmarking of out-of-hospital cardiac arrest (OHCA) performance between regions.

\section{METHODS}

This was a population-based cohort study of OHCA using data from the AusROC Australian and New Zealand OHCA Epistry over the period of 01 January 2015 to 31 December 2015. Backwards stepwise loglinear analysis was used to examine the relationship between key Utstein variables (arrest location, aetiology, witnessed status, bystander CPR, and cardiac arrest rhythm). For the loglinear analysis, cases were restricted to those with a presumed cardiac aetiology and with attempted resuscitation from EMS.

\section{RESULTS}

In 2015, there were 19,722 OHCA cases recorded in the Aus-ROC Epistry. Resuscitation was attempted by EMS in $48 \%$ of cases (range: $40 \%-68 \%$ ). For those who received attempted resuscitation, variation between ambulance

services was observed in unadjusted overall survival to hospital discharge or 30 days (range: $9 \%-17 \%$ ).

Loglinear analysis revealed four key interactions between key Utstein variables:

1. Location * witnessed status * arrest rhythm

2. Bystander CPR * arrest rhythm

3. Location * bystander CPR

4. Witnessed status * bystander CPR

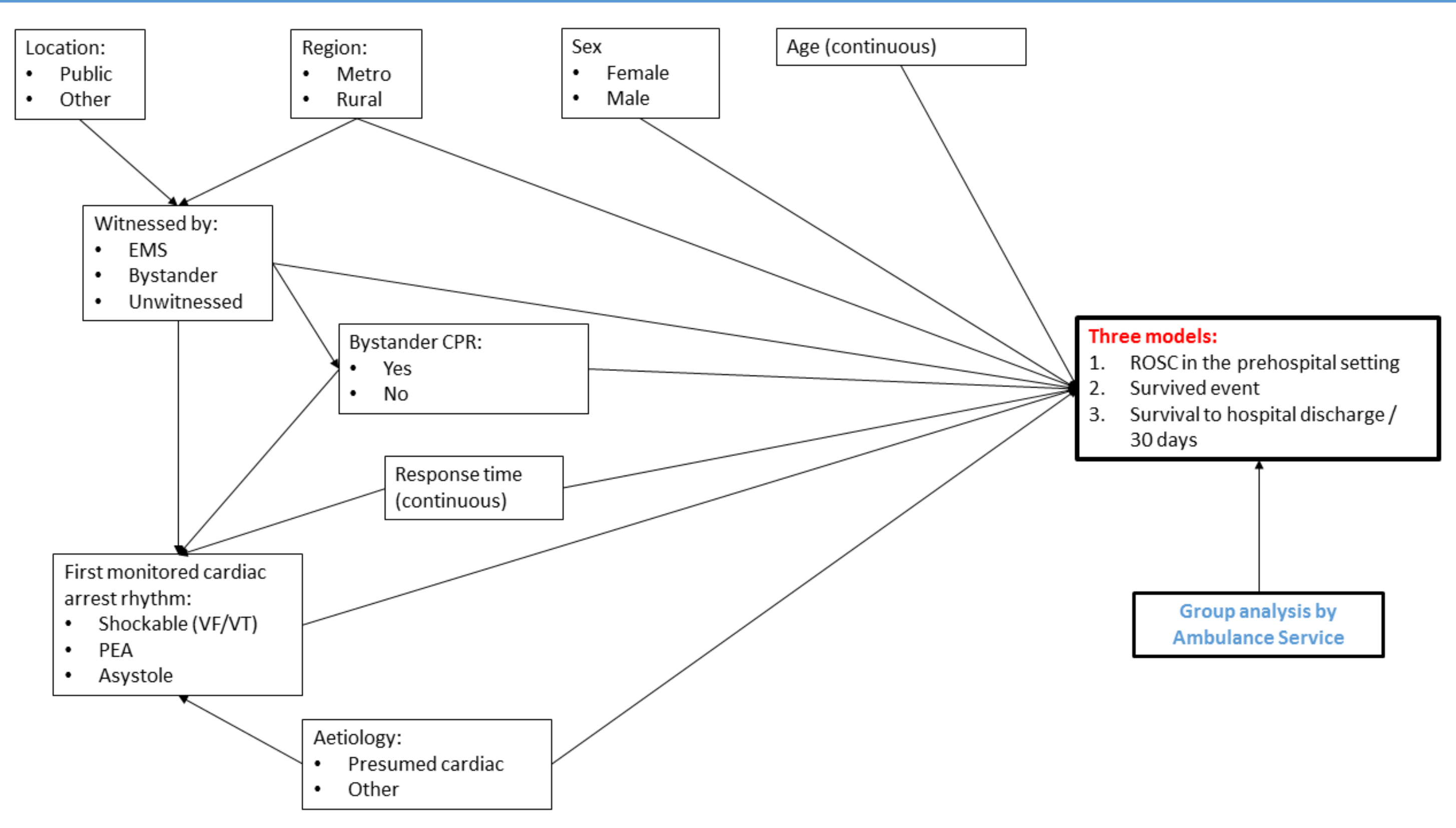

Figure 1: Proposed structural equation model demonstrating the inter-relationship between key OHCA variables

\section{CONCLUSIONS}

Given the significant variation in key Utstein variables between ambulance services, appropriate risk-adjusted modelling is required to account for this variation in underlying prognostic factors. As we have demonstrated, the interplay between these key factors is complex and multifactorial. This complexity is often not well accounted for in standard multivariable logistic regression models. We propose the use of structural equation models (Figure 1) to enable modelling the complex interplay between variables that will facilitate benchmarking of OHCA outcomes between regions. 